\title{
The SPECTRUM of the CURL OPERATOR on SPHERICALLY SYMMETRIC DOMAINS
}

\author{
Jason Cantarella, Dennis DeTurck, Herman Gluck and Mikhail Teytel
}

\begin{abstract}
This paper presents a mathematically complete derivation of the minimum-energy divergence-free vector fields of fixed helicity, defined on and tangent to the boundary of solid balls and spherical shells. These fields satisfy the equation $\nabla \times V=\lambda V$, where $\lambda$ is the eigenvalue of curl having smallest non-zero absolute value among such fields. It is shown that on the ball the energy-minimizers are the axially symmetric spheromak fields found by Woltjer and Chandrasekhar-Kendall, and on spherical shells they are spheromak-like fields. The geometry and topology of these minimum-energy fields, as well as of some higher-energy eigenfields, is illustrated.
\end{abstract}

Jason Cantarella

Department of Mathematics

University of Massachusetts

Amherst, MA 01003-4515

cantarel@math.umass.edu

Dennis DeTurck, Herman Gluck and Mikhail Teytel

Department of Mathematics

University of Pennsylvania

Philadelphia, PA 19104-6395

deturck@math . upenn . edu

gluck@math. upenn . edu

teytel@math.upenn.edu

PACS numbers:

- 02.30.Tb Mathematical methods in physics - operator theory

- 02.30.Jr Mathematical methods in physics - PDEs

- 47.10.+g Fluid dynamics - general theory

- 47.65.+a Magnetohydrodynamics and electrohydrodynamics

- 52.30.Bt MHD equilibria

- 52.30.-q Plasma flow; magnetohydrodynamics

- 52.55.Fa Tokamaks

- 52.55.Hc Stellerators, spheromaks, etc.

- 95.30.Qd Astrophysics: MHD and plasmas 


\section{Introduction}

The helicity of a smooth vector field $V$ defined on a domain $\Omega$ in 3-space was introduced by Woltjer ${ }^{1}$ in 1958 and named by Moffatt ${ }^{2}$ in 1969. It is the standard measure of the extent to which the field lines wrap and coil around one another, and is defined by the formula

$$
H(V)=\frac{1}{4 \pi} \int_{\Omega \times \Omega} V(x) \times V(y) \cdot \frac{x-y}{|x-y|^{3}} d\left(\operatorname{vol}_{x}\right) d\left(\operatorname{vol}_{y}\right) .
$$

Woltjer showed, in this same paper, that magnetic helicity and magnetic energy are both conserved in a non-dissipative plasma, and that an energy-minimizing magnetic field $V$ with fixed helicity, if it exists, must satisfy the equation $\nabla \times V=\lambda V$ for some constant $\lambda$ (and thus be a so-called constant- $\lambda$ force-free field). He also wrote that in a system in which the magnetic forces are dominant and in which there is a mechanism to dissipate the fluid motions, the force-free fields with constant $\lambda$ are the "natural end configurations".

In 1974, Taylor ${ }^{3,4}$ extended this idea by arguing that in a low-beta plasma (one in which magnetic forces are large compared to the hydrodynamic forces) confined in a vessel with highly conducting walls, the total magnetic helicity will be approximately conserved during the various magnetic reconnections that occur, and the conducting walls will act as a reasonably effective helicity escape barrier, while the magnetic energy of the plasma rapidly decays towards a minimum value. The resulting configuration can be found mathematically by assuming that the helicity remains constant while the energy is minimized.

Towards this end, we showed ${ }^{5,6,7,8}$ that among divergence-free vector fields which are tangent to the boundary of a given compact domain in 3-space, the energyminimizers for fixed helicity

- exist, are analytic in the interior of the domain, and are as differentiable at the boundary of the domain as is the boundary itself;

- satisfy an additional boundary condition which says that their circulation around any loop on the boundary must vanish if that loop bounds a surface exterior to the domain; 
- satisfy the equation $\nabla \times V=\lambda V$, with $\lambda$ having least possible absolute value among such fields.

The operator theoretic methods that we use were inspired by the work of $\mathrm{Arnold}^{9}$, and seem to provide a uniform and simple approach to finding and analyzing these energy-minimizing fields, as well as to proving their existence and determining their degree of differentiability. The fact that they are constant- $\lambda$ force-free fields was already known to Woltjer ${ }^{1}$ as mentioned above, who argued via a Lagrange-multiplier approach which assumed existence. The existence of these energy-minimizing fields was rigorously established by Laurence and Avellaneda ${ }^{10}$ in 1991, via a "constructive implicit function theorem", and was also analyzed by Yoshida and Giga ${ }^{11,12,13}$ in the early 1990s. The additional boundary condition stated above appears to be new.

In the case that all boundary components of the domain are simply connected (as is true for spherically symmetric domains), this additional boundary condition is automatically satisfied by all curl eigenfields which are tangent to the boundary. For in such a case, any loop on the boundary is itself the boundary of a portion of this surface; the circulation of $V$ around the loop equals the flux of $\nabla \times V$ through this surface-portion, and since $\nabla \times V=\lambda V$ which is tangent to the boundary, the flux is zero.

In this paper, we solve the equation $\nabla \times V=\lambda V$ with these boundary conditions on balls and spherical shells, prove that our set of solutions is complete, and identify the solutions with minimum eigenvalue. Our work confirms that the solutions of Chandrasekhar-Kendall ${ }^{14}$ and Woltjer ${ }^{15,1}$ on the ball form a complete set of solutions to the problem, and that the minimum eigenvalue fields are the usual spheromak fields as they asserted (see Sec. V, however, for some comments on their method). Moreover, we see how closely the minimum eigenvalue fields on spherical shells resemble the spheromak fields on balls.

Thus, we study the eigenvalue problem

$$
\nabla \times V=\lambda V
$$


for vector fields defined on a round ball in $R^{3}$, or a spherical shell (the domain between two concentric round spheres in $R^{3}$ ). The vector field $V$ must satisfy the additional conditions:

1. $V$ must be divergence-free, i.e., $\nabla \cdot V=0$ on the domain.

2. $V$ must be tangent to the boundary of the domain, i.e., if $\mathbf{n}$ is the outwardnormal vector to the boundary of the domain, then $V \cdot \mathbf{n}=0$ everywhere on the boundary.

By abuse of language, we will call vector fields that satisfy all these conditions "eigenfields of curl", and the corresponding eigenvalues "eigenvalues of curl".

We note that under these circumstances, the eigenvalue $\lambda=0$ cannot occur. That is because non-vanishing vector fields which are divergence-free, curl-free and tangent to the boundary of a compact domain $\Omega$ in $R^{3}$ can occur, according to the Hodge Decomposition Theorem ${ }^{8,16}$, only when the one-dimensional homology $H_{1}(\Omega ; R)$ of the domain is non-zero. We also note that a vector field $V$ which satisfies $\nabla \times V=\lambda V$ for some non-zero $\lambda$ is automatically divergence-free.

We prove the following results:

Theorem A. For the ball $B^{3}(b)$ of radius $b$, the eigenvalue of curl with least absolute value is $\lambda=4.4934 \ldots / b$, where the numerator is the first positive solution of the equation $x=\tan x$. It is an eigenvalue of multiplicity three, and its eigenfields are all images under rotations of $R^{3}$ of constant multiples of the one given in spherical coordinates by:

$$
V(r, \theta, \phi)=u(r, \theta) \hat{r}+v(r, \theta) \hat{\theta}+w(r, \theta) \hat{\phi}
$$

where $\hat{r}, \hat{\theta}$ and $\hat{\phi}$ are unit vector fields in the $r, \theta$ and $\phi$ directions, respectively, and

$$
\begin{gathered}
u(r, \theta)=\frac{2 \lambda}{r^{2}}\left(\frac{\lambda}{r} \sin (r / \lambda)-\cos (r / \lambda)\right) \cos \theta \\
v(r, \theta)=-\frac{1}{r}\left(\frac{\lambda}{r} \cos (r / \lambda)-\frac{\lambda^{2}}{r^{2}} \sin (r / \lambda)+\sin (r / \lambda)\right) \sin \theta
\end{gathered}
$$


SPECTRUM OF CURL ON SPHERICALLY SYMMETRIC DOMAINS

$$
w(r, \theta)=\frac{1}{r}\left(\frac{\lambda}{r} \sin (r / \lambda)-\cos (r / \lambda)\right) \sin \theta .
$$

Figure 1 is a picture of this vector field. It is axially symmetric and its integral curves fill up a family of concentric "tori", with a "core" closed orbit, features which are known ${ }^{17}$ to be typical of energy-minimizing, axisymmetric curl eigenfields. A special orbit, beginning at the south pole of the bounding sphere at time $-\infty$, proceeds vertically up the $z$-axis and reaches the north pole at time $+\infty$. Orbits on the bounding sphere start at the north pole at time $-\infty$ and proceed down lines of longitude to the south pole at time $+\infty$. There are two stationary points, one at each pole. Woltjer ${ }^{15}$ used this vector field to model the magnetic field in the Crab Nebula.

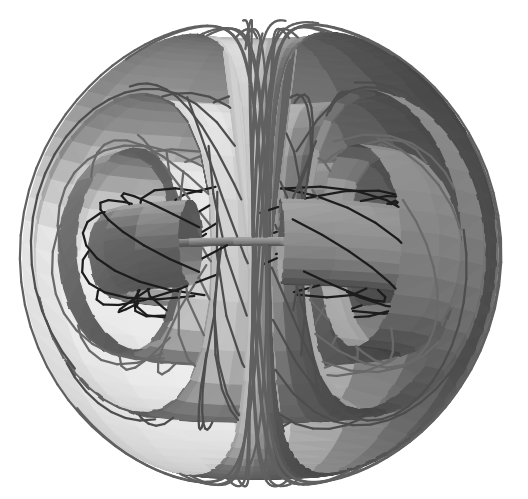

Figure 1. Integral curves and surfaces of the spheromak vector field on the ball

TheOREM B. For the spherical shell $B^{3}(a, b)$ of inner radius a and outer radius $b$, the eigenvalue of curl having least absolute value is $\lambda_{1}^{(1)}$, where $\lambda_{1}^{(1)}$ is the smallest of the infinite sequence of positive numbers $x_{k}$ that satisfy

$$
J_{\frac{3}{2}}(a x) Y_{\frac{3}{2}}(b x)-J_{\frac{3}{2}}(b x) Y_{\frac{3}{2}}(a x)=0
$$

(as a approaches zero, this reduces to the equation $x=\tan x$ of Theorem $A$ ). It is an eigenvalue of multiplicity three, and its eigenfields are all images under rotations of $R^{3}$ of constant multiples of the one given in spherical coordinates by:

$$
V(r, \theta, \phi)=u(r, \theta) \hat{r}+v(r, \theta) \hat{\theta}+w(r, \theta) \hat{\phi}
$$


where

$$
\begin{gathered}
u(r, \theta)=r^{-3 / 2}\left(c_{1} J_{\frac{3}{2}}\left(\lambda_{1}^{(1)} r\right)+c_{2} Y_{\frac{3}{2}}\left(\lambda_{1}^{(1)} r\right)\right) \cos \theta \\
v(r, \theta)=-\frac{1}{2 r} \frac{\partial}{\partial r}\left(\sqrt{r}\left(c_{1} J_{\frac{3}{2}}\left(\lambda_{1}^{(1)} r\right)+c_{2} Y_{\frac{3}{2}}\left(\lambda_{1}^{(1)} r\right)\right)\right) \sin \theta \\
w(r, \theta)=\frac{\lambda_{1}^{(1)}}{2 \sqrt{r}}\left(c_{1} J_{\frac{3}{2}}\left(\lambda_{1}^{(1)} r\right)+c_{2} Y_{\frac{3}{2}}\left(\lambda_{1}^{(1)} r\right)\right) \sin \theta
\end{gathered}
$$

This vector field is also axisymmetric and is qualitatively like the one on the ball, having a family of concentric tori as invariant surfaces, and exceptional orbits on both the inner and outer spherical boundaries. The invariant surfaces are pictured in Figure 2.

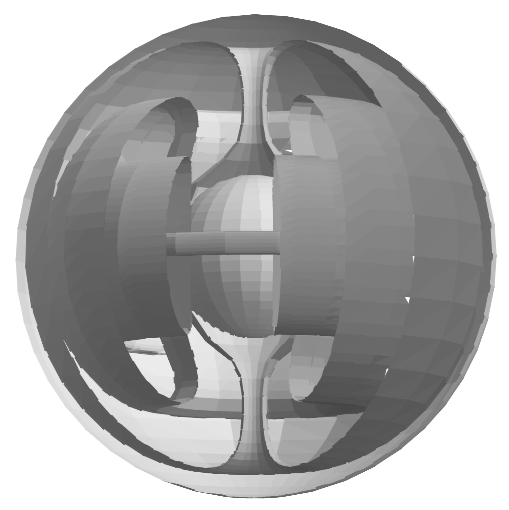

Figure 2. Integral surfaces of energy-minimizing vector field on a spherical shell.

An outline of this paper is as follows. Sec. II contains formulas for the curl and other expressions in spherical coordinates that will be used in the remainder of the paper. In Sec. III, we show that the radial component of any eigenfield of curl must satisfy an elliptic boundary value problem whose solutions are expressible in terms of eigenfunctions of the Laplace operator. Based on this observation, the other components of curl eigenfields are calculated in Sec. IV.

In Sec. V, we explain why our set of eigenfields is complete. Then we identify the eigenvalue of smallest absolute value in Sec. VI, and show that among spherically 
symmetric domains, the ball has the smallest such eigenvalue. Since this eigenvalue is the ratio of energy to helicity, it shows that the ball admits the least energy for given helicity among all spherically symmetric domains of a fixed volume. Finally, in Sec. VII, we examine some of the other eigenvalues and eigenfields.

\section{The curl operator in spherical coordinates.}

To fix our notation, we begin by reviewing how to write the curl operator and several related formulas in spherical coordinates. Throughout this paper, we take $r$, $\theta$ and $\phi$ to be the standard spherical coordinates. We let $\hat{r}=\frac{\partial}{\partial r}, \hat{\theta}=\frac{1}{r} \frac{\partial}{\partial \theta}$ and $\hat{\phi}=\frac{1}{r \sin \theta} \frac{\partial}{\partial \phi}$ be unit vector fields in the $r, \theta$ and $\phi$ directions respectively.

We will always consider a vector field $V(r, \theta, \phi)$ with components

$$
V(r, \theta, \phi)=u(r, \theta, \phi) \hat{r}+v(r, \theta, \phi) \hat{\theta}+w(r, \theta, \phi) \hat{\phi}
$$

For such a vector field, we have that

$$
\begin{aligned}
\nabla \times V=\frac{1}{r \sin \theta}\left(\frac{\partial}{\partial \theta}(\sin \theta w)-\frac{\partial v}{\partial \phi}\right) \hat{r} & +\frac{1}{r}\left(\frac{1}{\sin \theta} \frac{\partial u}{\partial \phi}-\frac{\partial}{\partial r}(r w)\right) \hat{\theta} \\
& +\frac{1}{r}\left(\frac{\partial}{\partial r}(r v)-\frac{\partial u}{\partial \theta}\right) \hat{\phi} .
\end{aligned}
$$

The eigenvalue equation $\nabla \times V=\lambda V$ thus reduces to a system of three partial differential equations:

$$
\begin{gathered}
\frac{1}{r \sin \theta}\left(\frac{\partial}{\partial \theta}(\sin \theta w)-\frac{\partial v}{\partial \phi}\right)=\lambda u \\
\frac{1}{r}\left(\frac{1}{\sin \theta} \frac{\partial u}{\partial \phi}-\frac{\partial}{\partial r}(r w)\right)=\lambda v \\
\frac{1}{r}\left(\frac{\partial}{\partial r}(r v)-\frac{\partial u}{\partial \theta}\right)=\lambda w .
\end{gathered}
$$

Since $\lambda \neq 0$, as mentioned earlier, the vector field $V$ is automatically divergence-free (by taking the divergence of both sides of $\nabla \times V=\lambda V$ and using the fact that the divergence of a curl is zero). Thus equations (2.1)-(2.3) imply

$$
\frac{1}{r^{2}} \frac{\partial}{\partial r}\left(r^{2} u\right)+\frac{1}{r \sin \theta} \frac{\partial}{\partial \theta}(\sin \theta v)+\frac{1}{r \sin \theta} \frac{\partial w}{\partial \phi}=0
$$


This is simply the equation $\nabla \cdot V=0$ in spherical coordinates.

The requirement that $V$ be tangent to the boundary $\partial \Omega$ of our domain $\Omega$ is equivalent to the condition

$$
u=0 \text { on } \partial \Omega \text {. }
$$

Because the square of the curl is the negative of the Laplacian for divergencefree vector fields, we will need the formula for the Laplacian of a scalar function in spherical coordinates. It is:

$$
\Delta f=\frac{1}{r^{2}} \frac{\partial}{\partial r}\left(r^{2} \frac{\partial f}{\partial r}\right)+\frac{1}{r^{2} \sin \theta} \frac{\partial}{\partial \theta}\left(\sin \theta \frac{\partial f}{\partial \theta}\right)+\frac{1}{r^{2} \sin ^{2} \theta} \frac{\partial^{2} f}{\partial \phi^{2}}
$$

It will also be helpful to have the formula for the gradient of a scalar function in spherical coordinates:

$$
\nabla f=\frac{\partial f}{\partial r} \hat{r}+\frac{1}{r} \frac{\partial f}{\partial \theta} \hat{\theta}+\frac{1}{r \sin \theta} \frac{\partial f}{\partial \phi} \hat{\phi}
$$

We need one more preliminary formula, for the Laplacian of the standard round metric induced on the sphere $S^{2}(r)$ of radius $r$ in Euclidean space. Because the Euclidean metric in $R^{3}$ is given in spherical coordinates by

$$
d s^{2}=d r^{2}+r^{2} d \theta^{2}+r^{2} \sin ^{2} \theta d \phi^{2}
$$

it is easy to see that the metric induced on $S^{2}(r)$ is

$$
d s_{r}^{2}=r^{2}\left(d \theta^{2}+\sin ^{2} \theta d \phi^{2}\right)
$$

where $r$ is taken to be a constant. The Laplacian with respect to this metric of a function $g$ defined only on $S^{2}(r)$ is then

$$
\Delta_{r}(g)=\frac{1}{r^{2}}\left(\frac{1}{\sin \theta} \frac{\partial}{\partial \theta}\left(\sin \theta \frac{\partial g}{\partial \theta}\right)+\frac{1}{\sin ^{2} \theta} \frac{\partial^{2} g}{\partial \phi^{2}}\right)
$$

\section{III. $\hat{r}$ component of the general solution}


Now we turn to solving the equation $\nabla \times V=\lambda V$ on the spherically symmetric domain $\Omega$. We are looking for three functions $u(r, \theta, \phi), v(r, \theta, \phi)$ and $w(r, \theta, \phi)$ that satisfy the system of three partial differential equations (2.1),(2.2) and (2.3). We also want $V$ to be tangent to the boundary of $\Omega$. This translates into boundary conditions on $u$ as follows: If $\Omega$ is the ball $B^{3}(b)$, then we require $u(0, \theta, \phi)$ to be bounded and $u(b, \theta, \phi)=0$. If $\Omega$ is the spherical shell $B^{3}(a, b)$, then we require that $u(a, \theta, \phi)=0$ and $u(b, \theta, \phi)=0$.

In this section we will concentrate on $u$, the $\hat{r}$ component of $V$. Our motivation comes from the fact that for divergence-free vector fields, we have $\nabla \times \nabla \times V=-\Delta V$. In rectangular coordinates, $\Delta V$ is simply the coordinate-wise Laplacian of $V$. But in spherical coordinates, this is no longer the case. We concentrate on $u$ because it is the "most rectangular" of the spherical coordinates (in that the integral curves of $\hat{r}$ are straight lines) and extract our second-order PDE for $u$ from the $\hat{r}$ component of $\nabla \times \nabla \times V=\lambda^{2} V$.

Proposition 1. Let $\Omega$ be the ball $B^{3}(b)$ or the spherical shell $B^{3}(a, b)$. If $u(r, \theta, \phi)$ is the $\hat{r}$ component of a divergence-free eigenfield $V$ of $\nabla \times V=\lambda V$ on $\Omega$, then $u$ must satisfy the equation

$$
\Delta u+\frac{2}{r} \frac{\partial u}{\partial r}+\frac{2}{r^{2}} u=-\lambda^{2} u
$$

together with the boundary conditions $u(0, \theta, \phi)$ is bounded and $u(b, \theta, \phi)=0$ if $\Omega=$ $B^{3}(b)$, or $u(a, \theta, \phi)=0$ and $u(b, \theta, \phi)=0$ if $\Omega=B^{3}(a, b)$.

Proof. The proposition follows from two observations about eigenfields of the curl operator. If $\nabla \times V=\lambda V$ and $\nabla \cdot V=0$, then $\Delta V=-\lambda^{2} V$. And for any vector field $V=u \hat{r}+v \hat{\theta}+w \hat{\phi}$ that satisfies $\Delta V=-\lambda^{2} V$ and $\nabla \cdot V=0$, we have that

$$
\Delta(r u)=-\lambda^{2}(r u)
$$


To see this, define the vector field $R=x \mathbf{i}+y \mathbf{j}+z \mathbf{k}=r \hat{r}$ and suppose that in rectangular coordinates $V=a \mathbf{i}+b \mathbf{j}+c \mathbf{k}$. Then $R \cdot V=r u$ and so

$$
\begin{aligned}
\Delta(r u) & =\Delta(R \cdot V)=\Delta(x a+y b+z c) \\
& =x \Delta a+y \Delta b+z \Delta c+2\left(\frac{\partial a}{\partial x}+\frac{\partial b}{\partial y}+\frac{\partial c}{\partial z}\right) \\
& =R \cdot \Delta V+2 \nabla \cdot V=-\lambda^{2} R \cdot V \\
& =-\lambda^{2}(r u) .
\end{aligned}
$$

We use formula (2.5) to calculate that $\Delta r=2 / r$ and of course $\nabla r=\hat{r}$; therefore

$$
\Delta(r u)=r \Delta u+2(\nabla r) \cdot(\nabla u)+u \Delta r=r \Delta u+2 \frac{\partial u}{\partial r}+\frac{2}{r} u=-\lambda^{2} r u
$$

Divide both sides by $r$ to obtain the equation in the proposition. The boundary conditions follow easily from the fact that $u=V \cdot n$ on the boundary.

This completes the proof of Proposition 1. Our task now is to solve the boundaryvalue problem for $u$.

Proposition 2. On the ball $B^{3}(b)$, the eigenvalues of equation (3.1) are given by the set $\left\{\lambda_{k}^{(n)}\right\}$ for $n=1,2,3, \ldots$ and $k=1,2,3, \ldots$, where the product $b \lambda_{k}^{(n)}$ is the $k$ th (positive) zero of the Bessel function $J_{n+\frac{1}{2}}(x)$. Each eigenvalue $\lambda_{k}^{(n)}$ has multiplicity $2 n+1$ with eigenfunctions given by:

$$
u(r, \theta, \phi)=r^{-3 / 2} J_{n+\frac{1}{2}}\left(\lambda_{k}^{(n)} r\right) P_{n}^{m}(\cos \theta)\left\{\begin{array}{c}
\cos m \phi \\
\sin m \phi
\end{array}\right\}
$$

where $m$ runs from 0 to $n \in\{1,2,3, \ldots\}$ (and of course we can only use the cosine solution for $m=0$ ).

Proposition 3. On the spherical shell $B^{3}(a, b)$, the eigenvalues and eigenfunctions of equation (3.1) are determined as follows: For every choice of $n \in\{0,1,2, \ldots\}$ and each $\lambda>0$, there are constants $c_{1}$ and $c_{2}$ such that:

$$
R(a)=c_{1} J_{n+\frac{1}{2}}(\lambda a)+c_{2} Y_{n+\frac{1}{2}}(\lambda a)=0
$$


and there is an infinite sequence of values of $\lambda$, which we label $\lambda_{k}^{(n)}$, such that

$$
R(b)=c_{1} J_{n+\frac{1}{2}}\left(\lambda_{k}^{(n)} b\right)+c_{2} Y_{n+\frac{1}{2}}\left(\lambda_{k}^{(n)} b\right)=0
$$

These values of $\lambda_{k}^{(n)}$ are our eigenvalues corresponding to the fixed value of $n$. The multiplicity of $\lambda_{k}^{(n)}$ is $2 n+1$, and we have

$$
u(r, \theta, \phi)=r^{-3 / 2}\left(c_{1 k}^{(n)} J_{n+\frac{1}{2}}\left(\lambda_{k}^{(n)} r\right)+c_{2 k}^{(n)} Y_{n+\frac{1}{2}}\left(\lambda_{k}^{(n)} r\right)\right) P_{n}^{m}(\cos \theta)\left\{\begin{array}{l}
\cos m \phi \\
\sin m \phi
\end{array}\right\}
$$

where $n \in\{1,2,3, \ldots\}, k \in\{1,2,3, \ldots\}$ and $m$ runs from 0 to $n$.

Proof of Propositions 2 and 3. According to the proof of Proposition 1, ru $(r, \theta, \phi)$ is an eigenfunction of the Laplacian with eigenvalue $-\lambda^{2}$. So we can begin by dividing the well-known expressions for eigenfunctions of the Laplacian in spherical coordinates by $r$ (see Stratton ${ }^{18}$, p. 404, eq. 22 for a classic reference). We find that all of the separable solutions of equation (3.1) are of the form:

$$
C_{\lambda m n} r^{-3 / 2}\left\{\begin{array}{c}
J_{n+\frac{1}{2}}(\lambda r) \\
Y_{n+\frac{1}{2}}(\lambda r)
\end{array}\right\} P_{n}^{m}(\cos \theta)\left\{\begin{array}{c}
\cos m \phi \\
\sin m \phi
\end{array}\right\}
$$

where we shall determine $\lambda$ from the boundary conditions, and thus far we have that $n \in\{0,1,2, \ldots\}$ and $m \in\{0,1,2, \ldots, n\}$. In this equation $J_{n+\frac{1}{2}}$ and $Y_{n+\frac{1}{2}}$ are Bessel functions of the first and second kinds of order $n+\frac{1}{2}$, and $P_{n}^{m}$ are associated Legendre functions.

We now turn to the issue of boundary conditions, which will discretize the set of $\lambda$ that can occur.

For the ball $B^{3}(b)$, we note that only Bessel functions of the first kind $J_{n+\frac{1}{2}}(\lambda r)$ may appear, and not the $Y_{n+\frac{1}{2}}$. This is because the $J$ 's remain bounded and the $Y^{\prime}$ 's become unbounded as $r$ approaches zero (in fact $Y_{\nu}(x)$ is asymptotic to $x^{-\nu}$ as $x$ approaches zero, see Lebedev ${ }^{19}$, p. 135). Therefore the $r$ part of the solution on the sphere must be $r^{-3 / 2} J_{n+\frac{1}{2}}(\lambda r)$. Also, since $J_{\nu}(x)$ is asymptotic to $x^{\nu}$ as $x$ approaches zero (see Lebedev ${ }^{19}$, p. 134), we see that the solution for $n=0$, which is $r^{-3 / 2} J_{1 / 2}(\lambda r)$, will be unbounded as $r \rightarrow 0$. This yields the conclusion of Proposition 2 . 
The situation for the spherical shell $B^{3}(a, b)$ is a little more complicated. For every choice of $n \in\{0,1,2, \ldots\}$ and each $\lambda>0$, there are constants $c_{1}$ and $c_{2}$ (unique up to scalar multiple of the vector $\left.\left[c_{1}, c_{2}\right]\right)$ such that:

$$
R(a)=c_{1} J_{n+\frac{1}{2}}(\lambda a)+c_{2} Y_{n+\frac{1}{2}}(\lambda a)=0 .
$$

This determines $R(r)$ up to a constant multiple. For most values of $\lambda$, we will have $R(b) \neq 0$. But there will be an infinite sequence of $\lambda$, which we label $\lambda_{k}^{(n)}$ as above, such that

$$
R(b)=c_{1} J_{n+\frac{1}{2}}\left(\lambda_{k}^{(n)} b\right)+c_{2} Y_{n+\frac{1}{2}}\left(\lambda_{k}^{(n)} b\right)=0 .
$$

These values of $\lambda_{k}^{(n)}$ will be our eigenvalues for curl corresponding to the fixed value of $n$. As in the case of the ball, the multiplicity of $\lambda_{k}^{(n)}$ is $2 n+1$. Also, as in the case of the ball, we may not choose $n=0$, although the reason for this is more complicated, and we will discuss it at the end of the next section. Our $\hat{r}$ components for the region between two concentric spheres are thus

$$
u(r, \theta, \phi)=r^{-3 / 2}\left(c_{1 k}^{(n)} J_{n+\frac{1}{2}}\left(\lambda_{k}^{(n)} r\right)+c_{2 k}^{(n)} Y_{n+\frac{1}{2}}\left(\lambda_{k}^{(n)} r\right)\right) P_{n}^{m}(\cos \theta)\left\{\begin{array}{c}
\cos m \phi \\
\sin m \phi
\end{array}\right\}
$$

where $n \in\{1,2,3, \ldots\}, k \in\{1,2,3, \ldots\}$ and $m$ runs from 0 to $n$ as before, proving Proposition 3 .

\section{An ordinary differential system for the $\hat{\theta}$ and $\hat{\phi}$ components}

Now we turn to finding the $\hat{\theta}$ and $\hat{\phi}$ components of our solution to $\nabla \times V=\lambda V$. We assume that we have in hand a function $u(r, \theta, \phi)$ that satisfies equation (3.1). Our first goal is to prove that given any such function $u$, there is at most one pair of functions $v$ and $w$ so that the vector $V=u \hat{r}+v \hat{\theta}+w \hat{\phi}$ satisfies $\nabla \times V=\lambda V$ (and $\nabla \cdot V=0$ in the case where $\lambda=0)$.

Proposition 4. Let $\Omega=B^{3}(a, b)$ (where a is allowed to be 0 ), and suppose that the function $u(r, \theta, \phi)$ is given. If $v_{1}(r, \theta, \phi), w_{1}(r, \theta, \phi)$ and $v_{2}(r, \theta, \phi), w_{2}(r, \theta, \phi)$ are two pairs of functions such that both $V_{1}=u \hat{r}+v_{1} \hat{\theta}+w_{1} \hat{\phi}$ and $V_{2}=u \hat{r}+v_{2} \hat{\theta}+w_{2} \hat{\phi}$ satisfy 
$\nabla \times V_{1}=\lambda V_{1}$ and $\nabla \times V_{2}=\lambda V_{2}$ (and $\nabla \cdot V_{1}=\nabla \cdot V_{2}=0$ in case $\lambda=0$ ), then it must be the case that $v_{1}=v_{2}$ and $w_{1}=w_{2}$.

Proof. Because $V_{1}$ and $V_{2}$ are eigenfields of the vector Laplace operator, as noted in the proof of Proposition 1, they are smooth on the interior of $\Omega$ by elliptic regularity. Set $f=v_{1}-v_{2}$ and $g=w_{1}-w_{2}$. Then the vector field $Q=V_{1}-V_{2}=f \hat{\theta}+g \hat{\phi}$ is also smooth on the interior of $\Omega$ and therefore its components relative to any orthonormal coordinate system are bounded. In particular, this holds for spherical coordinates, despite the singularities in the coordinate system along the $z$-axis. Furthermore, $Q$ satisfies $\nabla \times Q=\lambda Q$ and $\nabla \cdot Q=0$.

The $\hat{r}$ component of the equation $\nabla \times Q=\lambda Q$ is

$$
\frac{1}{r \sin \theta}\left(\frac{\partial}{\partial \theta}(\sin \theta g)-\frac{\partial f}{\partial \phi}\right)=0
$$

and

$$
\nabla \cdot Q=\frac{1}{r \sin \theta}\left(\frac{\partial}{\partial \theta}(\sin \theta f)+\frac{\partial g}{\partial \phi}\right)=0 .
$$

The first of these equations implies that

$$
\frac{\partial^{2}}{\partial \phi \partial \theta}(\sin \theta g)=\frac{\partial^{2} f}{\partial \phi^{2}}
$$

and the second implies that

$$
\frac{\partial^{2}}{\partial \phi \partial \theta}(\sin \theta g)=-\frac{\partial}{\partial \theta}\left(\sin \theta \frac{\partial}{\partial \theta}(\sin \theta f)\right) .
$$

Together, these last two equations imply that

$$
\frac{\partial}{\partial \theta}\left(\sin \theta \frac{\partial}{\partial \theta}(\sin \theta f)\right)+\frac{1}{\sin \theta} \frac{\partial^{2}}{\partial \phi^{2}}(\sin \theta f)=0
$$

We divide this equation by $r^{2} \sin \theta$ and recognize it (using formula (2.7) for the Laplacian on the sphere) as the condition $\Delta_{r}(\sin \theta f)=0$ for each value of $r$. Thus, the restriction of $\sin \theta f$ to any sphere centered at the origin is a harmonic function, and is hence constant on the sphere. Because $f$ must be bounded and continuous as remarked above, $\sin \theta f$ is zero at the north and south poles of the spheres. Therefore 
$f$ is identically zero. A similar argument shows that $g$ must be identically zero as well, proving the proposition.

Now we know that for each eigenfunction $u$ of equation (3.1) there can be at most one eigenfield $V$ of $\nabla \times V=\lambda V$ having $u$ as its $\hat{r}$ component. We now show that all of the eigenfunctions found in Propositions 2 and 3 give rise to eigenfields. To save notation, we will write $J_{\nu}(\lambda r)$ instead of $c_{1} J_{\nu}(\lambda r)+c_{2} Y_{\nu}(\lambda r)$ in the statement and proof of the following proposition. Also, where there are functions one atop the other and plus and minus signs one atop the other, only two solutions are represented: one must select all of the top choices or all of the bottom ones.

Proposition 5. Suppose $n \in\{1,2,3, \ldots\}$. Let $u$ be one of the $2 n+1$ eigenfunctions of equation (3.1) on $B^{3}(a, b)$ (where a is allowed to be zero in the case of the ball) corresponding to the eigenvalue $\lambda=\lambda_{k}^{(n)}$ of Proposition 2 or 3 , with $u(a, \theta, \phi)=$ $u(b, \theta, \phi)=0$ (or $u(0, \theta, \phi)$ is bounded in the case of the ball). Set $\nu=n+\frac{1}{2}$, choose $m \in\{0,1, \ldots, n\}$, and write

$$
u=\left(\nu^{2}-\frac{1}{4}\right) r^{-3 / 2} J_{\nu}(\lambda r) P_{n}^{m}(\cos \theta)\left\{\begin{array}{c}
\cos m \phi \\
\sin m \phi
\end{array}\right\}
$$

Then for

$$
v=\frac{1}{r} \frac{\partial}{\partial r}\left(\sqrt{r} J_{\nu}(\lambda r)\right) \frac{\partial}{\partial \theta} P_{n}^{m}(\cos \theta)\left\{\begin{array}{l}
\cos m \phi \\
\sin m \phi
\end{array}\right\} \mp \frac{\lambda m}{\sqrt{r}} J_{\nu}(\lambda r) \frac{P_{n}^{m}(\cos \theta)}{\sin \theta}\left\{\begin{array}{l}
\sin m \phi \\
\cos m \phi
\end{array}\right\}
$$

and

$$
w=\mp \frac{m}{r} \frac{\partial}{\partial r}\left(\sqrt{r} J_{\nu}(\lambda r)\right) \frac{P_{n}^{m}(\cos \theta)}{\sin \theta}\left\{\begin{array}{c}
\sin m \phi \\
\cos m \phi
\end{array}\right\}-\frac{\lambda}{\sqrt{r}} J_{\nu}(\lambda r) \frac{\partial}{\partial \theta} P_{n}^{m}(\cos \theta)\left\{\begin{array}{c}
\cos m \phi \\
\sin m \phi
\end{array}\right\}
$$

the vector field $V=u \hat{r}+v \hat{\theta}+w \hat{\phi}$ satisfies $\nabla \times V=\lambda V$ and $V \cdot n=0$ on the boundary of the domain.

Proof. One obvious way to prove this proposition is to check that $V$ as specified actually solves the equation $\nabla \times V=\lambda V$. On the other hand, this would give no clue 
SPECTRUM OF CURL ON SPHERICALLY SYMMETRIC DOMAINS

as to how $v$ and $w$ were calculated. So we give an indication of how we solved for $v$ and $w$. To begin, we assume that we have $u(r, \theta, \phi)$ as in the proposition, and set

$$
f=\frac{1}{\sin \theta} \frac{\partial u}{\partial \phi} \quad \text { and } \quad g=\frac{\partial u}{\partial \theta}
$$

Then the $\hat{\theta}$ and $\hat{\phi}$ components of $\nabla \times V=\lambda V$ become

$$
\frac{1}{r}\left(f-\frac{\partial}{\partial r}(r w)\right)=\lambda v \quad \text { and } \quad \frac{1}{r}\left(\frac{\partial}{\partial r}(r v)-g\right)=\lambda w .
$$

So we let $p=r v$ and $q=r w$ and note that these equations can be considered to be a system of two ordinary differential equations in $p$ and $q$ with $r$ as the independent variable, and parameters that depend on $\theta$ and $\phi$. The equations are

$$
p^{\prime}-\lambda q=g \quad \text { and } \quad q^{\prime}+\lambda p=f
$$

We need to find a particular solution of this constant-coefficient system of ODEs. We did so by a variation on the method of undetermined coefficients, expanding $p$ and $q$ as

$$
p=\sum_{\tau} a_{\tau} r^{1 / 2} J_{\tau}(\lambda r) \quad \text { and } \quad q=\sum_{\tau} b_{\tau} r^{1 / 2} J_{\tau}(\lambda r)
$$

where $\tau$ ranges over half-integers (i.e., numbers of the form $k+\frac{1}{2}$, where $k$ is an integer). We used several well-known identities for Bessel functions to show that all derivatives of $r^{1 / 2} J_{\tau}(\lambda r)$ can be expressed as linear combinations of terms of the same sort, for various values of $\tau$, thus giving us corresponding expansions for $p^{\prime}$ and $q^{\prime}$. We substituted the expansions for $p, q, p^{\prime}$ and $q^{\prime}$ into (4.2), and obtained a recurrence relation for the coefficients $a_{\tau}$ and $b_{\tau}$. We made the optimistic assumption that $a_{\tau}=b_{\tau}=0$ for all $\tau \leq \nu-2$, consistent with preliminiary calculations in the axially symmetric case, in order to get the recurrence started. We then solved recursively for the $a_{\tau}$ and $b_{\tau}$, and were surprised and delighted to find that all but three of these coefficients vanish, leaving us with finite sums for $p$ and $q$. We used our Bessel function identities once again to write $v=p / r$ and $w=q / r$ in the form given in the statement of Proposition 5.

Finally, we note that we do not have to worry about any other solutions of the system of ODEs from which we calculated $p$ and $q$, because the solutions we have 
already found are smooth, and by Proposition 4, they are the only possible smooth solutions. This completes the proof of Proposition 5.

We have thus calculated all the solutions of $\nabla \times V=\lambda V$ with the possible exception of solutions that come from Proposition 3 for $n=0$ on the domain $B^{3}(a, b)$. We now eliminate this possibility.

Proposition 6. There are no nonzero solutions of $\nabla \times V=\lambda V$ with $\nabla \cdot V=0$ having $\hat{r}$ component equal to a function of $r$ alone.

This will deal with the $n=0$ case because the eigenvalues $\lambda_{k}^{(0)}$ occur with multiplicity one, and because the Legendre function $P_{0}^{0}(\cos \theta)$ is a constant as is $\cos m \phi$ for $m=0$.

Proof. To begin the proof of Proposition 6, we return to the system (4.1) of ODEs for the $\hat{\theta}$ and $\hat{\phi}$ components of $V=u \hat{r}+v \hat{\theta}+w \hat{\phi}$, which are implied by the $\hat{\theta}$ and $\hat{\phi}$ components of $\nabla \times V=\lambda V$. Since $u$ is a function of $r$ alone, these components yield the system

$$
\begin{aligned}
& \frac{\partial(r w)}{\partial r}=-\lambda r v \\
& \frac{\partial(r v)}{\partial r}=\lambda r w
\end{aligned}
$$

These imply that

$$
\begin{aligned}
& v=\frac{c_{1}(\theta, \phi)}{r} \sin (\lambda r)-\frac{c_{2}(\theta, \phi)}{r} \cos (\lambda r) \\
& w=\frac{c_{1}(\theta, \phi)}{r} \cos (\lambda r)+\frac{c_{2}(\theta, \phi)}{r} \sin (\lambda r)
\end{aligned}
$$

where $c_{1}$ and $c_{2}$ are smooth functions on the (unit) sphere. The $\hat{r}$ component of $\nabla \times V=\lambda V$ multiplied by $r^{2}$ now becomes the equation:

$$
\begin{aligned}
\cos (\lambda r)\left(\frac{1}{\sin \theta}\right. & \left.\frac{\partial}{\partial \theta}\left(\sin \theta c_{1}\right)+\frac{1}{\sin \theta} \frac{\partial c_{2}}{\partial \phi}\right) \\
+ & \sin (\lambda r)\left(\frac{1}{\sin \theta} \frac{\partial}{\partial \theta}\left(\sin \theta c_{2}\right)-\frac{1}{\sin \theta} \frac{\partial c_{1}}{\partial \phi}\right)=\lambda r^{2} u(r)
\end{aligned}
$$

We substitute two different values of $r$ (that don't differ by $2 \pi / \lambda$ ) into the equation to see that the two expressions in parentheses must be constants, call them $\alpha$ and $\beta$. 
If we integrate the first of them over the (unit) sphere, we get that

$$
\int_{0}^{\pi} \int_{0}^{2 \pi}\left(\frac{\partial}{\partial \theta}\left(\sin \theta c_{1}\right)+\frac{\partial c_{2}}{\partial \phi}\right) d \phi d \theta=\int_{0}^{\pi} \int_{0}^{2 \pi} \alpha \sin \theta d \phi d \theta
$$

The integral on the left is zero because $\sin \theta c_{1}$ is zero for $\theta=0$ and $\theta=\pi$, and hence its $\theta$-derivative must $\theta$ - integrate to zero, and $c_{2}$ is $2 \pi$-periodic in $\phi$ so its $\phi$-derivative must $\phi$-integrate to zero. But the integral on the right is $4 \pi \alpha$, so $\alpha=0$. Similarly $\beta=0$. And since

$$
\alpha \cos (\lambda r)+\beta \sin (\lambda r)=\lambda r^{2} u
$$

with $\lambda \neq 0$, we have that $u=0$, in which case we must also have $v=w=0$ by Proposition 4. This completes the proof of Proposition 6.

Propositions 5 and 6 insure that we have found all of the eigenvalues and eigenfields for the equation $\nabla \times V=\lambda V$ that are divergence-free on $B^{3}(a, b)$ and tangent to the boundary.

\section{Completeness and comparison with Chandrasekhar-Kendall solutions}

Chandrasekhar and Kendall ${ }^{14}$ gave a method for constructing solutions of $\nabla \times V=$ $\lambda V$ from scalar eigenfunctions of the Laplace operator (solutions of $\Delta u=-\lambda^{2} u$ ) and simple (usually parallel) vector fields. We reviewed this technique ${ }^{6}$, and showed that, although it missed some of the eigenfields of $\nabla \times V=\lambda V$ on the flat solid torus, it did find all those which are divergence-free and tangent to the boundary.

The vector fields constructed in the preceding section constitute all of (or at least a basis for, since all of the eigenvalues are multiple) the eigenfields of $\nabla \times V=$ $\lambda V$ that are divergence-free and tangent to the boundary of the ball $B^{3}(b)$ or the domain $B^{3}(a, b)$ between two concentric spheres. This follows from the fact that we have complete sets of solutions of the Dirichlet problem for equation (3.1) for the $\hat{r}$ components of the eigenfields on both domains, and from Propositions 4 and 6, which show that for each solution $u$ of this equation, either there is a unique pair of functions $v$ and $w$ that complete $u$ to a solution $V=u \hat{r}+v \hat{\theta}+w \hat{\phi}$ of $\nabla \times V=\lambda V$ or else there is none. 
On the ball, all of these vector fields were found by Chandrasekhar and Kendall ${ }^{14}$, where there was no discussion of their completeness as solutions of the boundary-value problem. We have written our solutions so that they can be immediately recognized as the same as their equations (12), (13) and (14), although they have a misprint in equation (13). The new aspects of our work are to find the solutions on spherical shells as well as balls, and to demonstrate the completeness and energy-minimizing properties of our solutions.

\section{Energy minimizers}

To find the energy-minimizers on our domains $B^{3}(b)$ and $B^{3}(a, b)$, we have to find the eigenvalues of $\nabla \times V=\lambda V$ having smallest nonzero absolute value. Since we have in our possession all of the solutions of this equation, the task is not too difficult. We will prove the following:

Proposition 7. The smallest non-zero eigenvalue of $\nabla \times V=\lambda V$ on $B^{3}(b)$ and on $B^{3}(a, b)$ is $\lambda_{1}^{(1)}$, in the notation of Propositions 2 and 3.

Proof. Since the eigenvalues certainly satisfy

$$
\lambda_{1}^{(n)}<\lambda_{2}^{(n)}<\lambda_{3}^{(n)}<\cdots
$$

(since these are successive zeroes of some combination of Bessel functions), in order to prove the proposition, it is sufficient to show that

$$
\lambda_{1}^{(1)}<\lambda_{1}^{(2)}<\lambda_{1}^{(3)}<\cdots
$$

i.e., that $\lambda_{1}^{\left(n_{1}\right)}<\lambda_{1}^{\left(n_{2}\right)}$ if $n_{1}<n_{2}$.

Recall from Propositions 2 and 3 and their proofs that the eigenvalues $\lambda_{k}^{(n)}$ are the values of $\lambda$ for which the expression

$$
S_{\lambda}^{n}(r)=A J_{n+\frac{1}{2}}(\lambda r)+B Y_{n+\frac{1}{2}}(\lambda r)
$$

is nonzero but vanishes for both $r=a$ and $r=b$, where $A$ and $B$ are chosen so that $S_{\lambda}^{n}(a)=0$. For the ball, $a=0$, which implies that $B=0$ but we have $S_{\lambda}^{n}(0)=0$ in 
this case as well, since the function $u(r, \theta, \phi)$ is smooth and has an additional factor of $r^{-3 / 2}$. Being a linear combination of Bessel functions of order $n+\frac{1}{2}$ with argument $\lambda r$, the function $S_{\lambda}^{n}$ satisfies the differential equation

$$
r^{2} \frac{d^{2} S_{\lambda}^{n}}{d r^{2}}+r \frac{d S_{\lambda}^{n}}{d r}+\left(\lambda^{2} r^{2}-\left(n+\frac{1}{2}\right)^{2}\right) S_{\lambda}^{n}=0
$$

(see Stratton ${ }^{18}$, p. 404).

We shall use Sturm's comparison theorem ${ }^{20}$. Suppose $f(x)$ is a nonzero function that satisfies $f^{\prime \prime}+P(x) f=0$ with $f(a)=0$ and $g(x)$ is a nonzero function that satisfies $g^{\prime \prime}+Q(x) g=0$ with $g(a)=0$ (or we could assume $f^{\prime}(a)=g^{\prime}(a)=0$ ). Sturm's comparison theorem tells us that if $P(x) \geq Q(x)$ for all $x$, then on any interval containing $a$ and no (other) zeroes of $f(x)$, there can be no (other) zeroes of $g(x)$. Moreover, the "next" zero of $f(x)$ must be closer to $a$ than that of $g(x)$.

In order to apply Sturm's theorem to our problem, we need to make a change of variables to eliminate the first-order term in the differential equation. If we define $F(r)=\sqrt{r} S_{\lambda}^{n}(r)$, then $F$ will satisfy the equation

$$
\frac{d^{2} F}{d r^{2}}+\left(\lambda^{2}-\frac{n(n+1)}{r^{2}}\right) F=0
$$

Suppose $F_{1}^{(1)}$ is the solution of our equation that satisfies $F_{1}^{(1)}(x)>0$ for all $x$ between $a$ and $b$, and $F_{1}^{(1)}(b)=0$. So $F_{1}^{(1)}$ satisfies

$$
\frac{d^{2} F_{1}^{(1)}}{d r^{2}}+\left(\left(\lambda_{1}^{(1)}\right)^{2}-\frac{2}{r^{2}}\right) F_{1}^{(1)}=0 .
$$

For any $\lambda<\lambda_{1}^{(1)}$, and any $n>1$, we will certainly have

$$
\lambda^{2}-\frac{n(n+1)}{r^{2}}<\left(\lambda_{1}^{(1)}\right)^{2}-\frac{2}{r^{2}}
$$

Sturm's theorem tells us that no nonzero $F$ corresponding to such values of $\lambda$ and $n$ can satisfy $F(a)=F(b)=0$. Therefore all of the other eigenvalues of the boundaryvalue problem at hand must be larger than $\lambda_{1}^{(1)}$. Repeated application of the argument will prove the stronger inequality $\lambda_{1}^{\left(n_{1}\right)}<\lambda_{1}^{\left(n_{2}\right)}$ if $n_{1}<n_{2}$. This proves Proposition 8 . 
With the help of Maple, Table 1 contains values of $\lambda_{1}^{(1)}$ for $B^{3}(a, b)$, where $a=$ $\left(b^{3}-1\right)^{1 / 3}$ (so all of the domains have the same volume as the unit ball):

\begin{tabular}{|c|c|c||c|c|c|}
\hline$a$ & $b$ & $\lambda_{1}^{(1)}$ & $a$ & $b$ & $\lambda_{1}^{(1)}$ \\
\hline \hline 0 & 1 & 4.493409458 & 1.334200824 & 1.5 & 18.97447753 \\
.1442730320 & 1.001 & 4.568933565 & 1.912931183 & 2 & 36.08896092 \\
.2470322424 & 1.005 & 4.799466836 & 4.986630952 & 5 & 234.9901755 \\
.3117589910 & 1.01 & 5.033680133 & 9.996665555 & 10 & 942.1635951 \\
.5401839794 & 1.05 & 6.423856305 & 99.99996667 & 100 & 94257.20533 \\
.6917396417 & 1.1 & 7.858576168 & 999.9999997 & 1000 & 9424777.95775 \\
\hline
\end{tabular}

Table 1. Minimum eigenvalues $\lambda_{1}^{(1)}$ on $B^{3}(a, b)$.

From this data one can infer (correctly) that among all spherically symmetric domains with a given volume, the ball has the smallest value of $\lambda_{1}^{(1)}$, i.e., it supports the field of smallest energy for given helicity in a given volume. This is reminiscent of the situation for Dirichlet eigenvalues of the Laplace operator ${ }^{21}$, where the ball has the smallest possible $\lambda_{1}$ among all domains of the same volume in 3-space. On the other hand, we have shown ${ }^{22}$ that the ball does not support the field of smallest energy for given helicity among all (not necessarily spherically symmetric) domains of the same volume in 3-space.

All of the eigenfields corresponding to the first eigenvalue on spherically symmetric domains are axially symmetric. The multiplicity of the eigenvalue $\lambda_{1}^{(1)}$ is three, corresponding to three dimensions' worth of choices for the axis of symmetry. Maple plots of the three component functions $(u, v$ and $w$ in $V=u \hat{r}+v \hat{\theta}+w \hat{\phi})$ corresponding 
to $b=1.02$ on a constant- $\phi$ section of the domain $B^{3}(a, b)$ are in Figure 3 .

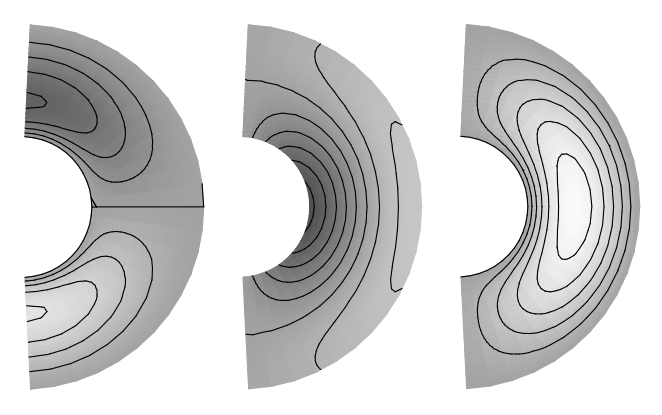

Figure 3. Level curves of the $\hat{r}, \hat{\theta}$ and $\hat{\phi}$ components of the energy-minimizing vector field on a constant- $\phi$ section of a spherical shell.

This yields the description in the introduction of the vector field with minimal energy on this spherical shell. The intersections of the invariant surfaces under the flow of the vector field with a constant- $\phi$ section of the domain are shown in the first plot in the next section.

\section{Other eigenfields}

It is interesting to examine the nature of the eigenfields of our problem for the curl operator corresponding to the other eigenvalues $\lambda_{k}^{(n)}$. We will look at the axially symmetric $(m=0)$ solutions corresponding to other eigenvalues. The topology of these solutions is essentially the same whether the domain is $B^{3}(b)$ or $B^{3}(a, b)$, so we will exhibit them only for one specific $\left.B^{(} a, b\right)$. Each is made up of several families of invariant tori ( $n k$ of them), half of which cycle in one direction around the $z$-axis and the other half cycle the other way. We look at plots of the intersections with the $\phi=0$ half-plane of the surfaces that are invariant under the flow defined by the eigenfields corresponding to $\lambda_{1}^{(1)}, \lambda_{2}^{(1)}, \lambda_{3}^{(1)}, \lambda_{1}^{(2)}, \lambda_{1}^{(3)}$ and $\lambda_{2}^{(2)}$ below. The first three plots are in Figure 4, and the last three are in Figure 5. 

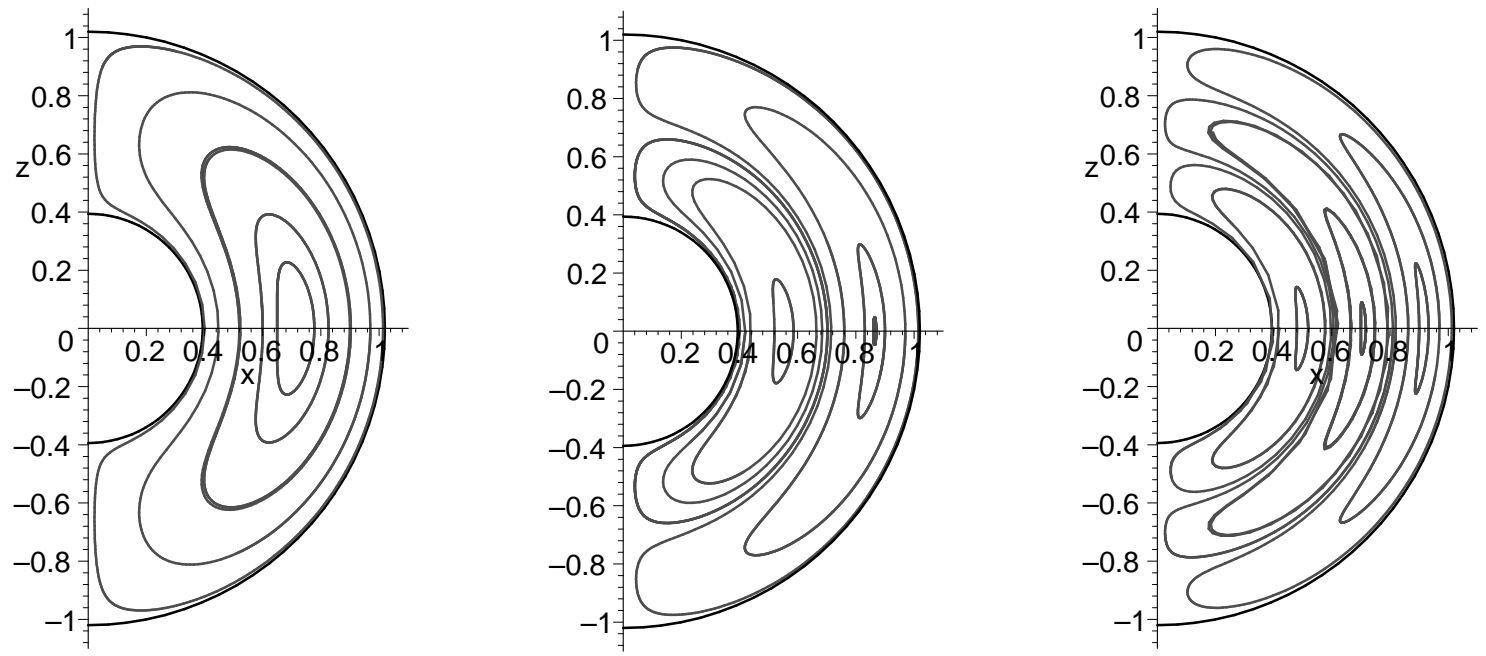

Figure 4. Traces of invariant surfaces for $\lambda_{1}^{(1)}, \lambda_{2}^{(1)}$ and $\lambda_{2}^{(1)}$ vector fields on a spherical shell.
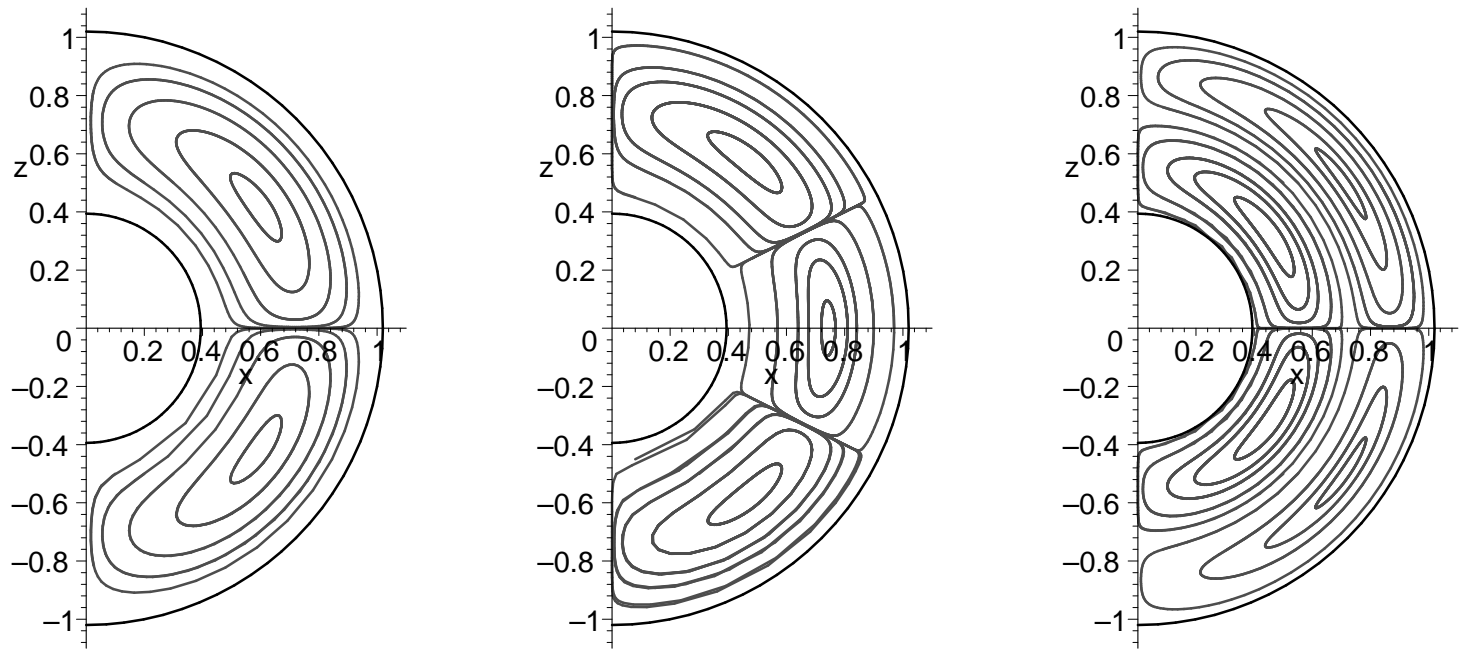

Figure 5. Traces of invariant surfaces for $\lambda_{1}^{(2)}, \lambda_{1}^{(3)}$ and $\lambda_{2}^{(2)}$ vector fields on a spherical shell.

We see that there are $k$ radial layers of $n$ families each. This topological arrangement is independent of the values of $a$ and $b$ in $B^{3}(a, b)$.

It is also interesting to calculate the eigenvalues to learn about the ordering of the energies of the eigenfields. We learn that as the spherical shell gets thinner and thinner, it is possible to expend less energy for given helicity by having a single radial layer with many families of tori ( $n$ large and $k=1$ ) rather than to have even two 
layers with a single family each $(n=1, k=2)$. Tables $2,3,4$ and 5 report the eigenvalues of curl for various $B(a, b)$. The values of $a$ and $b$ have been chosen so that the volumes of all of the domains are the same as the volume of the unit ball.

\begin{tabular}{|c||c|c|c|c|c|}
\hline & $n=1$ & $n=2$ & $n=3$ & $n=4$ & $n=5$ \\
\hline \hline$k=1$ & 4.49341 & 5.76346 & 6.98792 & 8.18256 & 9.35581 \\
$k=2$ & 7.72525 & 9.09501 & 10.4171 & 11.7949 & \\
$k=3$ & 10.9041 & 12.3229 & 13.6980 & 15.0397 & \\
$k=4$ & 14.0662 & 15.5146 & 16.9236 & & \\
$k=5$ & 17.2208 & 18.6890 & 20.1218 & & \\
$k=6$ & 20.3713 & 21.8539 & 23.3042 & & \\
\hline
\end{tabular}

Table 2. Values of $\lambda_{k}^{(n)}$ on the ball $B^{3}(1)$.

\begin{tabular}{|c||c|c|c|c|c|c|}
\hline & $n=1$ & $n=2$ & $n=3$ & $n=4$ & $n=5$ & $n=6$ \\
\hline \hline$k=1$ & 5.03368 & 5.92554 & 6.99240 & 8.12397 & 9.26946 & 10.4104 \\
$k=2$ & 9.32168 & 9.93620 & 10.7867 & & & \\
$k=3$ & 13.7236 & & & & & \\
$k=4$ & 18.1695 & & & & & \\
\hline
\end{tabular}

Table 3. Values of $\lambda_{k}^{(n)}$ on the shell $B^{3}(0.312,1.01)$.

\begin{tabular}{|c||c|c|c|c|c|c|c|c|}
\hline & $n=1$ & $n=2$ & $n=3$ & $n=4$ & $n=5$ & $n=6$ & $n=11$ & $n=12$ \\
\hline \hline$k=1$ & 7.85858 & 8.17541 & 8.62800 & 9.19535 & 9.85647 & 10.5924 & 14.9312 & 15.8707 \\
$k=2$ & 15.4746 & & & & & & & \\
\hline
\end{tabular}

Table 4. Values of $\lambda_{k}^{(n)}$ on the shell $B^{3}(0.692,1.1)$.

\begin{tabular}{|c||c|c|c|c|c|c|c|c|}
\hline & $n=1$ & $n=2$ & $n=3$ & $n=20$ & $n=100$ & $n=121$ & $n=122$ & $n=125$ \\
\hline \hline$k=1$ & 36.0890 & 36.1034 & 36.1251 & 37.5717 & 62.7739 & 71.8208 & 72.2631 & 73.5952 \\
$k=2$ & 72.1671 & 72.1743 & & & & & & \\
\hline
\end{tabular}

Table 5. Values of $\lambda_{k}^{(n)}$ on the shell $B^{3}(1.913,2)$. 


\section{Summary and Conclusions}

We have given a complete proof that every eigenfield of curl (equivalently, constant$\lambda$ force free field) which is divergence-free and tangent to the boundary of a spherically symmetric domain in 3-space is one of the fields described in Propositions 2, 3 and 5; that the only possible values for $\lambda$ are the $\lambda_{k}^{(n)}$; and that $\lambda_{1}^{(1)}$ is the least among them (Proposition 7).

These facts, together with our previous work ${ }^{7,8}$ on helicity and energy, allow us to conclude that the Taylor state for a low-beta plasma in a spherically symmetric vessel with highly conducting walls must be the spheromak field derived by ChandrasekharKendall $^{14}$ and Woltjer ${ }^{1,15}$ in the case of a solid ball, and the spheromak-like fields derived in this paper in the case of a spherical shell.

We have also observed that the topology of the field with eigenvalue $\lambda_{k}^{(n)}$ is similar

on all the spherical shells $B(a, b)$, even though the ordering of the $\lambda_{k}^{(n)}$ depends on $a$ and $b$ (except for $\lambda_{1}^{(1)}$ ).

In addition, we proved that the eigenvalue $\lambda_{1}^{(1)}$ on the ball is minimal among all the eigenvalues on spherically symmetric domains of the same volume. However ${ }^{22}$, there are other domains (not spherically symmetric) of equal volume on which the minimal eigenvalue is smaller than that on the ball. 


\section{REFERENCES}

1 L. Woltjer, A theorem on force-free magnetic fields, Proc. Nat. Acad. Sci. USA 44 (1958) 489-491.

2 H.K. Moffatt, The degree of knottedness of tangled vortex lines, J. Fluid Mech. 35 (1969) 117-129 and 159, 359-378.

3 J.B. Taylor, Relaxation of toroidal plasma and generation of reversed magnetic field, Phys. Rev. Lett., 33 (1974) 1139-1141.

4 J.B. Taylor, Relaxation and magnetic reconnection in plasmas, Rev. Mod. Phys., $58: 3$ (1986) 741-763

5 J. Cantarella, D. DeTurck and H. Gluck, Upper bounds for the writhing of knots and the helicity of vector fields, preprint, University of Pennsylvania, March 1997; to appear in Proceedings of the Conference in Honor of the 70th Birthday of Joan Birman, edited by J. Gilman, X-S. Lin and W. Menasco, International Press, AMS/IP Series on Advanced Mathematics (2000).

6 J. Cantarella, D. DeTurck and H. Gluck, The spectrum of the curl operator on the flat torus, preprint, University of Pennsylvania, March 1997; submitted to J. Fluid Mech.

7 J. Cantarella, D. DeTurck and H. Gluck, The Biot-Savart operator for application to knot theory, fluid mechanics and plasma physics, preprint, University of Pennsylvania, December 1997; submitted to J. Math. Phys.

8 J. Cantarella, D. DeTurck, H. Gluck and M. Teytel, Influence of geometry and topology on helicity, Magnetic Helicity in Space and Laboratory Plasmas, M. Brown, R. Canfield and A. Pevtsov (eds), Geophysical Monograph 111, American Geophysical Union (1999) 17-24.

9 V.I. Arnold, The asymptotic Hopf invariant and its applications, Proc. Summer School in Differential Equations, Erevan, Armenian SSR Academy of Sciences (1974); English translation in Selecta Math. Sov. 5(4)(1986) 327-345. 
10 P. Laurence and M. Avellaneda, On Woltjer's variational principle for force-free fields, J. Math. Phys. 32(5) (1991) 1240-1253.

11 Z. Yoshida and Y. Giga, Remarks on spectra of operator rot, Math. Z. 204 (1990) 235-245.

12 Z. Yoshida, Discrete eigenstates of plasmas described by the Chandrasekhar-Kendall functions, Progress of Theoretical Phys. 86(1) (1991) 45-55.

13 Z. Yoshida, Eigenfunction expansions associated with the curl derivatives in cylindrical geometries: Completeness of Chandrasekhar-Kendall eigenfunctions, J. Math. Phys. 33(4) (1992) 1252-1256.

14 S. Chandrasekhar and P.C. Kendall, On force-free magnetic fields, Astrophysical Journal 126 (1957) 457-460.

15 L. Woltjer, The Crab Nebula, Bull. Astr. Netherlands 14 (1958) 39-80.

16 J. Cantarella, D. DeTurck and H. Gluck, Hodge decomposition of vector fields on bounded domains in 3-space, preprint, University of Pennsylvania, December 1997.

17 J. Cantarella, Topological structure of stable plasma flows, Ph.D. Thesis, University of Pennsylvania, 1999.

18 J.A. Stratton, Electromagnetic Theory, first edition, McGraw-Hill, 1941.

19 N.N. Lebedev, Special Functions and their Applications, Dover Publications, 1972.

20 G.F. Simmons, Differential Equations with Applications and Historical Notes, second edition, McGraw-Hill, 1991.

21 G. Polya and S. Szego, Isoperimetric Inequalities in Mathematical Physics, Princeton, N.J.; Princeton University Press, 1951.

22 J. Cantarella, D. DeTurck, H. Gluck and M. Teytel, Isoperimetric problems for the helicity of vector fields and the Biot-Savart and curl operators, preprint, University of Pennsylvania, November 1998; to appear in J. Math. Phys. (May 2000). 